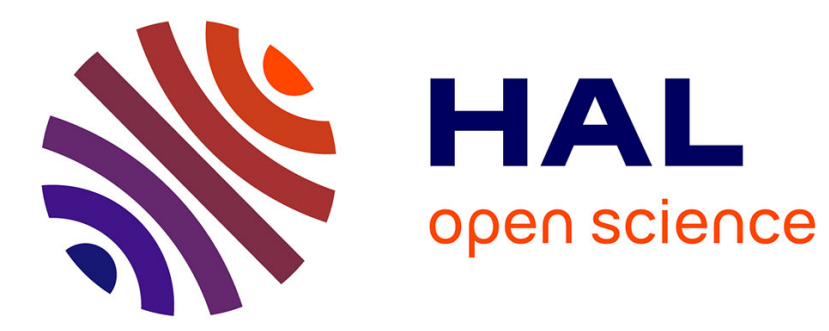

\title{
Modélisation de la température rectale post-mortem en environnement thermique variable
}

\author{
Damien Charabidze, Alexandre Veremme, Gildas Morvan, Valery Hedouin, \\ Didier Gosset
}

\section{- To cite this version:}

Damien Charabidze, Alexandre Veremme, Gildas Morvan, Valery Hedouin, Didier Gosset. Modélisation de la température rectale post-mortem en environnement thermique variable. La Revue de Médecine Légale, 2010, 1 (2), pp.61 - 65. 10.1016/j.medleg.2010.07.002 hal-03331906

\author{
HAL Id: hal-03331906 \\ https://hal.science/hal-03331906
}

Submitted on 24 Sep 2021

HAL is a multi-disciplinary open access archive for the deposit and dissemination of scientific research documents, whether they are published or not. The documents may come from teaching and research institutions in France or abroad, or from public or private research centers.
L'archive ouverte pluridisciplinaire HAL, est destinée au dépôt et à la diffusion de documents scientifiques de niveau recherche, publiés ou non, émanant des établissements d'enseignement et de recherche français ou étrangers, des laboratoires publics ou privés. 
Disponible en ligne sur<smiles>C1C2C3CC2C13</smiles>

0

www.sciencedirect.com
Elsevier Masson France

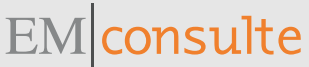

www.em-consulte.com

\title{
Modélisation de la température rectale post-mortem en environnement thermique variable
}

\section{Postmortem rectal temperature modelisation in fluctuating thermal environment}

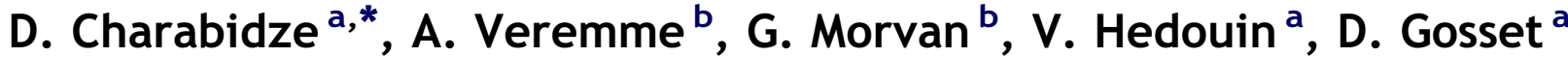 \\ a Institut médico-légal, université Lille Nord de France, 59000 Lille, France \\ b Pôle ingénierie et sciences du vivant (ISV), HEI, 13, rue de Toul, 59046 Lille, France
}

\section{MOTS CLÉS}

Température rectale ; Intervalle post-mortem ; Modélisation ; Inertie thermique ; Datation du décès ; Nomogramme de Henssge

\section{KEYWORDS}

Rectal temperature; Post-mortem interval; Modelisation;

Thermal inertia;

Death datation;

Hennsge's nomogram

\begin{abstract}
Résumé La mesure de la température corporelle est un des principaux critères de détermination de l'intervalle post-mortem (IPM). Après le décès, la température du corps s'aligne progressivement sur la température ambiante : cette évolution est régie par des lois de transferts thermiques connues. Un certain nombre de méthodes, parmi lesquelles le nomogramme de Henssge, se basent sur cette relation afin d'estimer l'IPM. Cependant, lorsque le corps se trouve à température ambiante variable, aucune méthode ne permet de prédire l'évolution de sa température interne. Ce travail présente les résultats d'un modèle permettant de simuler l'évolution de la température rectale d'un corps lorsque la température de son environnement varie. Les données obtenues grâce à ce modèle ont été comparées aux estimations d'IPM obtenues grâce au nomogramme de Henssge afin de tester la validité de celui-ci dans le cas d'un corps soumis à une température ambiante variable. Les résultats indiquent que le nomogramme de Henssge (et plus généralement les méthodes basées sur les données de décroissance thermique) ne peut être utilisé lorsque la température ambiante varie, même faiblement $\left(4^{\circ} \mathrm{C}\right.$ sur 24 heures).
\end{abstract}

(C) 2010 Elsevier Masson SAS. Tous droits réservés.

Summary The measure of the rectal temperature is one of the main post-mortem interval (PMI) estimation tool. After the death, the body temperature decreases until reaching the ambient temperature. This evolution is well known and governed by heat transfer laws. Several methods, as the Henssge's nomogram, use this thermal indicator to estimate the PMI. However, when the body environment temperature varies, no method allows to predict the evolution of the internal temperature. This work presents the results of a computer model allowing the simulation of human body thermal inertia, and evolution of the post-mortem rectal temperature in response to variable ambient temperatures. In order to test Henssge's nomogram validity in case of body at variable ambient temperature, model data were compared to PMI estimation obtained using the nomogram. Results indicate that the rectal temperature can not be use if the environment temperature vary, even if these variations have low amplitude $\left(4{ }^{\circ} \mathrm{C}\right.$ in $\left.24 \mathrm{~h}\right)$. (C) 2010 Elsevier Masson SAS. All rights reserved.

\footnotetext{
* Auteur correspondant.

Adresse e-mail : damien@forenseek.org (D. Charabidze).
} 


\section{Introduction}

La détermination de l'intervalle post-mortem (IPM) est une étape clef de l'expertise médico-légale. Quotidiennement, les praticiens sont amenés à estimer l'heure ou la date de la mort lors des levées de corps : leurs conclusions sont fondamentales sur le plan judiciaire. Pour répondre au mieux à cette question majeure, les médecins légistes ont à leur disposition différentes méthodes d'expertise qu'ils peuvent confronter et mutualiser. Certaines sont principalement qualitatives, comme la méthode de Vibert [16], et d'autres quantitatives, comme la mesure de la température corporelle ou de certaines constantes physiologiques $[2,4,6,8]$. Comparativement, l'évolution de la température rectale, hépatique ou tympanique constitue le critère le plus utilisé et un des plus fiables pour estimer l'heure de la mort $[2,12,13]$.

La cinétique de refroidissement d'un corps est relativement bien connue et a fait l'objet de nombreuses études détaillées [4,6,7,10,11]. La formule de Knight, fréquemment utilisée pour estimer l'IPM, se base sur une formulation très simple de cette décroissance thermique [3]. Un autre modèle, plus complexe et plus précis, a été établi par Henssge d'après les formules de Marshall, puis étalonné à partir d'une série de mesures réalisées sur 39 corps [4]. II se base sur une fonction bi-exponentielle pour décrire l'évolution de la température rectale entre le moment du décès et sa stabilisation sur la température de l'environnement. L'estimation de I'IPM repose ainsi sur deux paramètres d'entrée principaux : la température rectale et la température ambiante. Un cœfficient peut être utilisé pour pondérer cette relation en fonction de la dissipation thermique liée à l'environnement immédiat du cadavre (vent, vêtements, etc.) [5]. Fréquemment utilisé, le modèle de Henssge présente pourtant un certain nombre de limites. Outre sa gamme de validité assez restreinte (poids de 50 à $80 \mathrm{~kg}$, température constante) et l'absence de certains paramètres (taille et position du corps), son application est limitée au calcul de la température rectale. Une méthode alternative s'appuie sur la mesure de la température tympanique, dont la décroissance est plus stable et constitue donc un meilleur indicateur $[2,14]$. Bien que la pertinence de cette méthode ait été démontrée expérimentalement, elle reste délicate à mettre en œuvre et est, dans les faits, moins fréquemment employée que la température rectale [3,12,13].

Dans tous les cas, ces méthodes ne sont applicables que durant la phase de décroissance thermique et dans le cas de corps à température environnementale constante. Cependant, Althaus et Henssge [1] proposent une méthodologie permettant d'intégrer l'existence de fortes baisses de la température ambiante dans le calcul de l'IPM. Les auteurs indiquent également qu'en cas de variations progressives de la température du milieu, la température moyenne peut être utilisée « dans la majorité des cas » [1]. En revanche, leur étude conclut à l'impossibilité d'intégrer à l'analyse l'effet d'une augmentation brusque de la température ambiante.

En réponse à ces manques, un modèle basé sur une modélisation des flux de chaleur (principe des éléments finis) a été proposé par Mall et Eisenmenger [10]. Cette approche simule l'évolution la température post-mortem en subdivisant le corps en infimes éléments géométriques, chacun évoluant selon une série de lois thermiques propres.
Elle permet donc de connaître par interpolation la température en tous points, et autorise en théorie l'intégration de températures extérieures variables. Cependant, le niveau de précision du modèle requiert une parfaite description de l'environnement du corps, notamment concernant les transferts énergétiques par radiations, très difficiles à obtenir en pratique. De plus, ce travail a été réalisé dans un contexte commercial, et le programme n'est donc pas libre d'accès.

Bien que l'estimation de l'IPM grâce à la mesure (unique) de la température corporelle soit donc normalement strictement limitée aux corps à température environnementale constante, il n'est pas rare que ces méthodes soient appliquées dans le cas de corps découverts en extérieur, donc à température ambiante variable. Le simulateur présenté dans la suite de ce travail a été développé afin de modéliser l'évolution en différents points de la température postmortem. Après validation expérimentale, les prédictions de ce modèle ont été utilisées afin de tester l'effet de variations de la température environnementale sur l'évolution de la température rectale. Cette méthodologie permet ainsi, sans recourir à des mesures directes, de simuler in silico l'évolution de la température d'un corps en réponse à diverses variations thermiques de son environnement. Elle contourne ainsi les problèmes éthiques et techniques soulevés par la réalisation de longs (plusieurs jours) enregistrements thermiques post-mortem en conditions contrôlées sur des corps humains [9]. Ce modèle a ensuite été utilisé afin de tester les possibilités d'utilisation du nomogramme de Henssge lorsque la température de l'environnement du corps n'est pas constante.

\section{Matériel et méthode}

\section{Principe des automates cellulaires et modélisation du système}

Un réseau d'automates cellulaires peut être considéré comme une grille dont chacune des cases peut adopter, à un instant donné, un état dépendant de ses caractéristiques propres et de celles de son voisinage [15]. Le temps et l'espace y étant discrétisés (i.e. discontinus), l'état d'une case au temps $t$ dépendra de son état et de celui de son voisinage au temps $t-1$. À chaque pas de temps, les mêmes règles sont appliquées simultanément à toutes les cases, produisant ainsi une évolution de la grille.

Dans cette étude, le corps humain a été représenté en deux dimensions selon une coupe latérale (corps vu de face). Les cases ont été définies selon deux types principaux : fluide (environnement) ou solide (corps). Les cases périphériques correspondent ainsi à des zones de peau (en contact avec les cases de l'environnement et les autres cases du corps) tandis que les zones internes (muscles, viscères, etc.) sont uniquement en contact avec d'autres zones du corps. Les dimensions utilisées respectent les proportions classiques d'un corps, le nombre de cases (chacune mesurant $4 \mathrm{~cm} \times 4 \mathrm{~cm}$ ) variant selon la taille du cadavre. Les proportions des différents tissus ont été affectées d'après la littérature comme suit : masse musculaire : $35 \%$ de la masse corporelle totale, masse grasse : $15 \%$, masse viscérale : $25 \%$, masse osseuse : $15 \%$, peau : $7 \%$, cerveau : $3 \%$. 
Chaque case est définie par des caractéristiques physiques statiques (i.e. fixées) comme la masse, la taille et la chaleur massique (paramètre spécifique au type de tissus). Les constantes ont été déterminées d'après les valeurs publiées par Werner et Buse [17]. Pour un corps nu, la température est initialisée au moment du décès à $27^{\circ} \mathrm{C}$ au niveau de la surface de la peau et des extrémités, à $37,2^{\circ} \mathrm{C}$ au centre du tronc, $35^{\circ} \mathrm{C}$ pour les muscles et $36,5^{\circ} \mathrm{C}$ pour le cerveau [4]. La température d'une case de l'environnement peut être fixe (simulation à température ambiante stable) ou dynamique (température ambiante variable).

\section{Règles de transfert thermique}

Pour une case c en contact avec un fluide, la loi de Newton donne l'évolution de sa température suite à un échange par convection :

$T c(t)=T e+(T c(0)-T e) * \exp (-h * S * t)$

où Tc est la température $\left(\mathrm{en}^{\circ} \mathrm{C}\right)$ de la cellule au temps $t, \operatorname{Tc}(0)$ sa température initiale, Te la température de l'environnement de la cellule, $h$, le coefficient d'échange thermique (W.m-2. $\left.{ }^{\circ} \mathrm{C}-1\right), \mathrm{S}$, la surface du corps en contact avec l'environnement $\left(\mathrm{m}^{2}\right)$ et $t$, le temps (s) (Veremme et al., 2008). De la même manière, on détermine l'évolution de la température d'une case $c$ en contact avec un solide e grâce à l'équation de conduction :

$T c(t)=T e-(T e-T c(0)) * \exp \left(\frac{-t}{k}\right)$

où $k$ est le coefficient de conductivité (W.m-1.C-1).

Le transfert de chaleur par rayonnement n'a pas été intégré : il est le plus souvent négligeable (sauf exposition directe à un fort ensoleillement) et nécessite des données très détaillées (intensité du rayonnement, angle d'incidence, etc.) qui ne sont généralement pas disponibles. La taille d'une case étant négligeable devant celle du corps, la température de chacune des cases peut être calculée comme résultant de la moyenne des échanges thermiques réalisés avec son environnement immédiat ( 8 voisines). Pour passer de $t$ à $t+1$, chaque case applique donc l'algorithme suivant :
- parcourir l'ensemble des températures des voisines ;

- pour chacune des voisines, appliquer la loi thermique adaptée en fonction du type de celle-ci (fluide ou solide : convection ou conduction). Pour chaque échange, une case néglige les échanges de chaleur déjà réalisés avec ses autres voisines;

- faire la moyenne des températures issues de l'échange avec ses huit voisines pour obtenir la température à $t+1$.

\section{Validation}

Afin de valider le comportement du modèle, la température rectale simulée par l'automate cellulaire a, dans un premier temps, été confrontée à celle prédite par l'équation de Henssge (elle-même validée expérimentalement) dans le cas d'un cadavre humain se trouvant à une température constante de $20^{\circ} \mathrm{C}$ durant 200 heures consécutives au décès. La différence maximale enregistrée entre les valeurs prédites par le modèle de Henssge et l'automate cellulaire varie de $0,09{ }^{\circ} \mathrm{C}$ à $0,42{ }^{\circ} \mathrm{C}$ pour des corps allant de 50 à $80 \mathrm{~kg}$.

Le comportement du modèle a ensuite été confronté à des mesures effectuées en conditions semi-contrôlées (température ambiante variable) sur un cadavre de porc (Sus scrofa domesticus). Le cadavre d'un jeune mâle de $30,5 \mathrm{~kg}$ (euthanasié dans le cadre d'un projet de recherche médicale) a été équipé d'une sonde thermique (Testo $645, \pm 0,1^{\circ} \mathrm{C}$ ) insérée au niveau rectal. Une sonde témoin identique, placée à $10 \mathrm{~cm}$ du cadavre, a été utilisée pour enregistrer la température ambiante toutes les minutes. Le cadavre a été déposé dans une vaste pièce non meublée et non chauffée. Les fenêtres étaient ouvertes et équipées de moustiquaires pour empêcher toute colonisation par les insectes nécrophages. Les enregistrements ont débuté le 04 avril 2007 et ont été stoppés le 10 avril 2007. Les données enregistrées par la sonde témoin (température ambiante) ont ensuite été utilisées pour réaliser une simulation de l'évolution de la température rectale de ce cadavre (Fig. 1). En raison de la morphologie particulière au cadavre de porc, la structure de l'automate cellulaire a été modifiée sous une forme
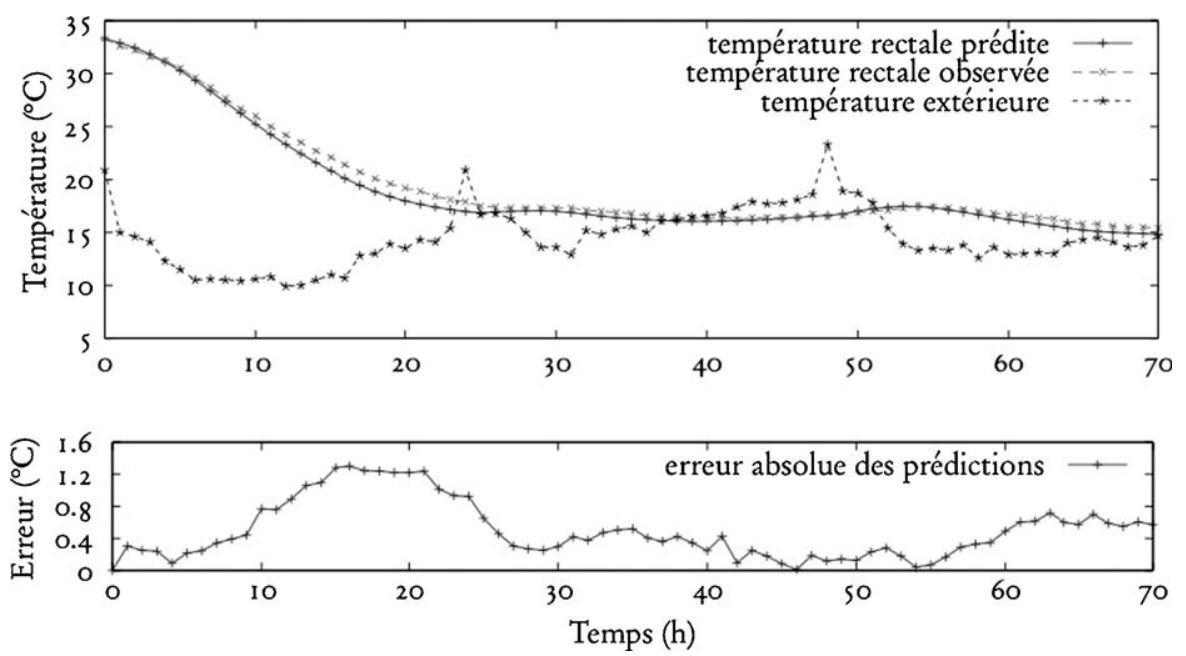

Figure 1 Évolution de la température rectale enregistrée et simulée d'un cadavre de porc de 30,5 kg placé à température ambiante variable. 

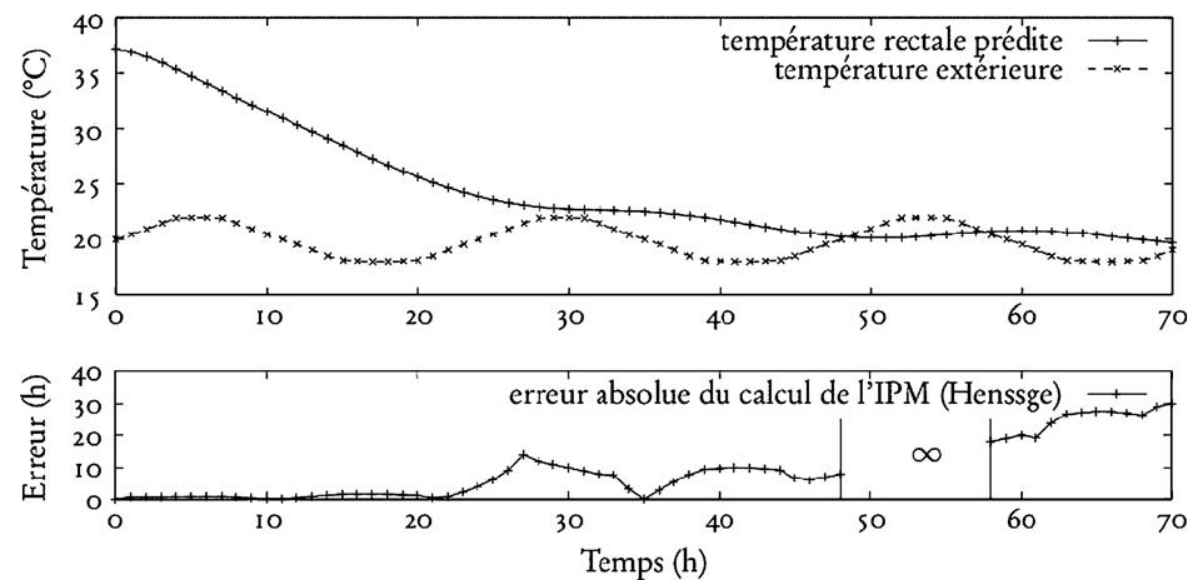

Figure 2 Simulation de la température rectale d'un cadavre humain $(1,70 \mathrm{~m}, 70 \mathrm{~kg})$ sous un régime de température cyclique (période 24 heures, amplitude $4{ }^{\circ} \mathrm{C}$, représenté en pointillés). La température rectale correspondante calculée par l'automate cellulaire est représentée en trait plein. Le second cadre (bas) du graphique représente l'erreur entre la datation fournie par le nomogramme de Henssge sur la base de ces données et l'IPM réel (IPM réel - IPM estimé, en heures et en valeur absolue).

assimilable à un rectangle de dimensions équivalentes à celles du corps $(90 * 40 \mathrm{~cm})$.

Une simulation a ensuite été réalisée afin de visualiser en conditions de température variable l'évolution de la température rectale post-mortem (corps de $70 \mathrm{~kg}, 1,70 \mathrm{~m}$, nu allongé au sol). Le régime de température ambiante testé présente un cycle oscillant sur une période de vingt-quatre heures avec une amplitude de $4{ }^{\circ} \mathrm{C}$. La température ambiante et la température du corps relevées au moment de la découverte du corps sont utilisées afin de calculer un IPM grâce à la formule de Henssge [4]. On confronte ensuite cette estimation à l'IPM réel indiqué par le simulateur.

\section{Résultats}

La comparaison à température ambiante constante des données du simulateurs avec celles fournies par l'équation de Henssge indique des résultats statistiquement identiques : ce premier test permet donc indirectement de valider les prévisions fournies par l'automate cellulaire. Cette démarche, déjà employée par Mall et Eisenmenger [10] pour la validation de leur modèle, apporte ainsi une première confirmation des prédictions de l'automate cellulaire. La confrontation des mesures expérimentales et des températures simulées dans le cas du cadavre de porc placé à température ambiante indique également une excellente concordance des deux jeux de données (test de corrélation de Pearson, alpha $=0,01$ ) (Fig. 1). L'écart maximum enregistré entre les températures prédites et observées ne dépasse pas $1,5^{\circ} \mathrm{C}$. On observe suite à la phase de décroissance initiale que la température interne reste sensiblement constante et comprise entre $15^{\circ} \mathrm{C}$ et $20^{\circ} \mathrm{C}$, tandis que la température ambiante varie fortement. On peut également noter que conjointement à cet effet d'amortissement, l'inertie du cadavre engendre une réponse thermique retardée. Il faut ainsi plusieurs heures pour qu'une modification de la température environnementale se répercute avec une intensité moindre au sein des tissus. Ce phénomène est parfaitement reproduit par le modèle d'automates cellulaires. Ces résultats confirment ainsi la validité prédictive du modèle développé (Fig. 1).
Les prédictions de l'automate cellulaire confirmées expérimentalement, celui-ci a été utilisé comme référence afin de tester l'effet de variations de la température ambiante sur l'estimation de l'IPM via la température rectale. On se place ici dans le cas d'un médecin légiste arrivant sur le site de découverte d'un corps, et se référant à la température rectale afin d'estimer l'IPM. Le corps au sol, nu, pèse environ $70 \mathrm{~kg}$ pour $1,70 \mathrm{~m}$. Cependant, la température ambiante n'est pas constante, et présente des variations de $\pm 2{ }^{\circ} \mathrm{C}$ sur une période de 24 heures. Cette variation est difficilement décelable (sauf enregistrement), mais va pourtant induire une modification de la cinétique de refroidissement du corps (Fig. 2), et ainsi engendrer une erreur dans l'estimation de l'IPM.

La température ambiante et la température rectale sont relevées par le légiste, qui se réfère au nomogramme de Henssge pour dater le décès. La différence entre la datation obtenue sur la base de la méthode de Henssge et l'IPM réel est représentée sur la Fig. 2. L'utilisation du nomogramme permet une bonne estimation de l'IPM pendant les vingt premières heures consécutives au décès, puis diffère significativement de l'IPM réel (automate cellulaire). Une erreur d'estimation de presque 15 heures est ainsi obtenue seulement 27 heures après le décès.

\section{Discussion}

Bien qu'il soit nécessaire de confirmer expérimentalement le modèle sur des corps humains [9], les résultats des expériences de validation confirment la qualité prédictive de l'automate cellulaire. Ainsi, la validation expérimentale réalisée avec un modèle animal (cadavre de porc) indique une excellente concordance des températures simulées et enregistrées, ce qui confirme que le modèle est capable de prédire l'évolution de la température post-mortem d'un corps lorsque la température extérieure varie. Sur cette base, le modèle a été utilisé afin de simuler l'effet de faibles variations de la température ambiante sur la cinétique de refroidissement d'un corps et l'estimation de I'IPM. Le régime de température testé correspond à un 
environnement extérieur présentant des variations thermiques de faible amplitude $\left(4^{\circ} \mathrm{C}\right)$ sur une période d'oscillation de vingt-quatre heures. Sans enregistrement, de telles variations sont difficilement décelables : un observateur pourra donc facilement postuler par erreur que la température ambiante est constante.

Notre modèle indique cependant que de telles oscillations ont un effet direct et non négligeable sur la cinétique thermique post-mortem. Du fait de son inertie, le corps réagit de manière différée à ces variations de la température ambiante : la température mesurée au niveau rectal présente de faibles oscillations en décalage (environ 10 heures) de celles de l'environnement. Ce phénomène peut avoir d'importantes conséquences sur l'estimation de l'IPM. En effet, bien que de très nombreuses méthodes alternatives existent, la mesure de la température rectale et l'utilisation du nomogramme de Henssge restent très fréquemment employées pour dater le décès. Dans le cas de figure présenté ici, la variabilité de la température ambiante et son effet sur la cinétique thermique post-mortem engendrent d'importantes erreurs dans l'estimation de l'IPM dès 22 heures après le décès. Ainsi, 27 heures après la mort, la température rectale atteint $23{ }^{\circ} \mathrm{C}$ tandis que la température ambiante est de $21,5^{\circ} \mathrm{C}$. L'utilisation du nomogramme conduit alors à un IPM de 42 heures, soit une surestimation de 14 heures environ. Il apparaît donc clairement que l'effet des variations environnementales rend inapplicable l'utilisation de méthodes telles que le nomogramme de Henssge, basées sur la décroissance thermique post-mortem.

La validité de méthodes telles que la mesure de la température hépatique ou de la température tympanique dans un tel cas de figure n'a pas été testée ici, celles-ci étant principalement appliquées dans le cas d'IPM courts (inférieurs à une journée) [2,7]. Il est cependant très probable que les variations de la température de l'environnement affectent encore plus fortement ces organes, rendant de fait également ces méthodes inapplicables.

\section{Conclusion}

La modélisation par automates cellulaires permet de reproduire le comportement thermique d'un corps. Un des principaux avantages de cette méthode est son utilisation à température ambiante variable, qui permet de simuler l'évolution de la température d'un corps situé en extérieur. Ce travail permet également de disposer d'un outil de simulation numérique bien plus aisé à employer que le recours à des expériences de terrain sur modèle animal, et a fortiori humain. Enfin, contrairement aux autres modèles existants, il est simple d'utilisation et libre d'accès, et ne nécessite pas de disposer de données extrêmement détaillées pour réaliser des simulations [10].

Après validation expérimentale, les prédictions de ce modèle informatique ont été utilisées afin de tester la validité du nomogramme de Henssge dans le cas d'un corps à température ambiante variable. Les résultats indiquent que la méthode de Henssge ne peut être utilisée dans le cas de corps trouvés dans un environnement dont la température varie, même avec une amplitude de seulement $4{ }^{\circ} \mathrm{C}$ (période de 24 heures).

\section{Conflit d'intérêt}

Damien Charabidze, chercheur au sein de l'Institut de médecine légale (université Lille 2 ), certifie par la présente que les auteurs de l'article intitulé « Modélisation de la température rectale post-mortem en environnement thermique variable » devant être publié dans la revue de médecine légale n'ont pas de conflits d'intérêts.

\section{Remerciements}

Les auteurs tiennent à remercier les Docteurs Y. Delannoy et C. Houssaye, ainsi que Monsieur D. Dupont pour leur aide et leurs conseils. Cette recherche a été réalisée avec le soutien financier de la Fondation Norbert Segard.

\section{Références}

[1] Althaus L, Henssge $C$. Rectal temperature time of death nomogram: sudden change of ambient temperature. Forensic Sci Int 1999;99:171-8.

[2] Baccino E, De Saint-Martin L, Schuliar Y, Guilloteau P, Le Rhun $\mathrm{M}$, Morin JF, et al. Outer ear temperature and time of death. Forensic Sci Int 1996;832:133-46.

[3] Beauthier JP. Traité de médecine légale; 2007: 837p.

[4] Henssge C. Death time estimation in case work. The rectal temperature time of death nomogram. Forensic Sci Int 1988; $38: 209-36$

[5] Henssge C. - Rectal temperature time of death nomogram: dependence of corrective factors on the body weight under stronger thermic insulation conditions. Forensic Sci Int 1992; 54:51-66.

[6] Henssge C, Madea B. Estimation of the time since death. Forensic Sci Int 2007;165:182-4.

[7] Kaliszan M, Hauser R, Kaliszan R, Wiczling P, Buczyñski J, Penkowski $M$. Verification of the exponential model of body temperature decrease after death in pigs. Exp Physiol 2005;90(5):727-38.

[8] Kanawaku Y, Kanetake J, Komiya A, Maruyama S, Funayama M. Computer simulation for postmortem cooling processes in the outer ear. Leg Med 2007;9(2):55-62.

[9] Kanetake J, Kanawaku Y, Funayama M. Automatic continuous monitoring of rectal temperature using a button-type thermo data logger. Leg Med 2006;8:226-30.

[10] Mall G, Eisenmenger W. Estimation of the time since death by heat-flow finite-element model. Legal Med 2005;7 (1-14):69-80.

[11] Marshall T, Hoare F. Estimating the time of death - the rectal cooling after death and its mathematical expression. J Forensic Sci 1962;7:56-81.

[12] Rutty GN. Concerning the paper by Baccino et al. Outer ear temperature and time of death. Forensic Sci Int 1997;87(2): $171-2$.

[13] Rutty GN. The estimation of the time since death using temperatures recorded from the external auditory canal. Forensic Sci Med Pathol 2005;1(1):41-51.

[14] Simonsen J, Voigt J, Jeppesen N. Determination of the time of death by continuous post-mortem temperature measurements. Med Sci Law 1977;17(2):112-22.

[15] Veremme A, Dupont D, Morvan G, Jolly D, Charabidze D. Modélisation de la température d'un corps par automates cellulaires. Paris: MOSIM; 2008.

[16] Vibert. Précis de médecine légale; 1890: $792 \mathrm{p}$.

[17] Werner J, Buse M. Temperature profiles with respect toinhomogeneity and geometry of the human body. Appl Physiol 1988;65:1110-8. 